\title{
Vulnerability assessment methodology for Swiss road network
}

\section{Journal Article}

Author(s):

Erath, Alex; Birdsall, James; Axhausen, Kay W. (D); Hajdin, Rade

Publication date:

2009-01

Permanent link:

https://doi.org/10.3929/ethz-b-000019517

Rights / license:

In Copyright - Non-Commercial Use Permitted

Originally published in:

Transportation Research Record 2137, https://doi.org/10.3141/2137-13 


\title{
Vulnerability Assessment Methodology for Swiss Road Network
}

\author{
Alex Erath, James Birdsall, Kay W. Axhausen, and Rade Hajdin
}

This paper presents a methodology that incorporates vulnerability to natural hazards into current infrastructure management systems. The paper is mainly concerned with presenting the methodology applied to assess the transport-related consequences of link failures, including congestion effects on a networkwide scale. Four possible demand shifts caused by single link failures can be expected: detours, shifts in mode choice, shifts in destination choice, and trip-activity suppression. The paper demonstrates that detours are by far the predominant demand reaction. Hence, the quantification of detour-based principal consequences is the main focus. The main challenge was to overcome the calculation time intensity of this equilibrium-based approach. Since demand shift effects were assumed to be spatially restricted around the failed link, subnetworks were used, that is, limited sections of the complete network that were cut out, including their internal and transit demands. The resulting failures turned out to be consistent with those that involved the full network, even for links with long path distances or long detours. On the basis of computed consequences of link failures and on link parameters, a statistical model was developed to reveal and quantify the main factors defining transportrelated consequences. Furthermore, the findings highlight potential gains, including rail networks, mode shifts, and destination choice shifts in network vulnerability assessments.

Infrastructure systems, such as transportation infrastructure systems, are composed of links connecting geographically dispersed communities, towns, and cities. When these systems operate as designed, they form the foundation upon which commerce, trade, and the serviced communities can flourish. But when the availability of these systems is jeopardized by gradual deterioration (e.g., corrosioninduced deterioration) or natural hazards (e.g., avalanche-induced link failure), the communities they service can likewise suffer. Over the past 20 years great strides have been made to address gradual deterioration of infrastructure objects (e.g., roads, bridges, tunnels). Given the scale of today's transport network, infrastructure management systems (IMSs) like PONTIS (1) or KUBA (2) have been developed to collate inspection data, model and predict future deterioration processes, and develop optimal infrastructure management approaches. However, the management of potential infrastructure failures due to natural hazard has not enjoyed a comprehensive or systemwide management perspective. The most common approach

A. Erath and K. W. Axhausen, Institute for Transport Planning and Systems (IVT), ETH Zurich, CH-8093 Zurich, Switzerland. J. Birdsall and R. Hajdin, Infrastructure Management Consultants LLC, Signaustrasse 14, CH-8008 Zurich, Switzerland. Corresponding author: A. Erath, erath@ivt.baug.ethz.ch.

Transportation Research Record: Journal of the Transportation Research Board, No. 2137, Transportation Research Board of the National Academies, Washington, D.C., 2009, pp. 118-126.

DOI: $10.3141 / 2137-13$ is to conduct localized or regional transportation natural hazard risk assessment and mitigation projects. These activities follow natural hazard events and result in localized management and mitigation approaches. A number of large-scale systematic risk assessment initiatives-including Risk Map Germany (3) and Riskscape New Zealand (4) — are under development, but only one platform — the Hazards United States Multi-Hazard Federal Emergency Management Agency (5)—has been implemented for systematically assessing risk from a national viewpoint. However, to integrate the natural hazards risk management into already existing management systems, one has not only to quantify the failure probability of a given infrastructure object to a given natural hazard but also to determine the resulting post-failure economic consequences.

Although in transport literature the term "vulnerability" is usually used to describe the transport-related consequences like additional travel times, distances, or decrease in accessibility we argue that vulnerability has to be considered as a combination of the probability of occurrence of a given hazard, the resistance of the infrastructure against it, and its consequences to transport.

This paper is organized as follows. The next section presents the recent approaches to quantify transport-related link failure consequences and builds the basis of the methodology development presented in the following section. The discussion of the results also includes an analysis of the accuracy of the applied approach. Given the enormous computational intensity and high input data quality required, the results were used to derive a statistical model to describe network failure consequences more straightforwardly, which is presented in the section on the statistical model. The paper ends with the conclusions and the needs for further research.

\section{LITERATURE}

The assessment of transport network failure consequences has attracted significant attention recently. The main focus of the relatively new notion is to assess not only the actual state of transportation infrastructure but also to assess the impact of a network deterioration to the community. The increasing frequency of natural hazards due to global warming (6), recent collapses of transportation links, for example, those that occurred in New Orleans and Switzerland because of natural hazards, but also the threat of terrorist attacks enhance the relevance of the topic. Nevertheless, the research community has not reached a common definition of link failure-induced transportation-related consequences. Several definitions by various authors provide different perspectives on transportation-related consequences including Berdica (7), Taylor and D'Este (8), Knoop et al. (9), and Matisziw et al. (10). However, they all assess the impact of infrastructure failure, though with different measures and methodologies: For instance Bell (11) and Bell and Cassir (12) have used a game 
theory approach and formulated the problem as a two-player, noncooperative, zero-sum game envisaging between a router seeking a least-cost path, and a virtual network tester seeking to maximize transportation consequences by severing one link at a time. This approach was applied to identify the most vulnerable network elements. However, when link costs are assumed to be traffic-dependent, which they are, such a methodology is very computationally intensive and only applicable on small networks. Besides Monte Carlo simulation (13) and Minimum Cut Set (14), approaches that incorporate both the demand and supply side of traffic assignment models were used recently for the assessment. They differ mainly in the type of traffic assignment used: Jenelius et al. (15) neglect the traffic dependency of travel times; as in the Swedish case, most parts of the country are only sparsely populated, and link capacity plays only a minor role in the analysis, with congestion therefore becoming only a minor problem as result of link failures. This might be a reasonable assumption for spatially disperse countries, but Knoop et al. (9) showed for the case of the Rotterdam metro region the need to include capacity constraints when analyzing road network failure consequences in more densely populated areas.

However, a major constraint of the use of traffic assignment models for analyzing transport-related failures is the computational intensity. As each link failure scenario has to be calculated separately and the calculation time for one equilibrium assignment for the Swiss network with over 60,758 directed links and 3,110 zones, using a rather loose stop criterion and hence fast computation times, takes about $40 \mathrm{~min}$, it is obvious that alternative approaches have to be addressed. This problem has also been identified by the research community, and some researchers have subsequently attempted to describe and forecast the vulnerable parts of a network with various indicators (16). These indicators include different measures of volume and volumecapacity ratio, the number of paths over a link, and spillback figures. These indicators were assessed on correlation between each other and rank order against the results of the full assignment. Unfortunately the results had no comprehensive explanatory power; the rank order test, particularly, lacked the necessary significance. Scott et al. (17) calculated the additional travel time due to link failure in three generic transport networks using Wardrop's user equilibrium principles (18) and named it the network robustness index (NRI). The intention was to prove that it could be more efficient to provide additional route links and transportation capacity not only at known bottlenecks, but also at vulnerable link failures because of missing alternative paths. This research showed some significant correlation between the volume-capacity ratio and the NRI, but reveal, as do Knoop et al. (16), important differences between the selections of the most vulnerable links by the different measures when they are assessed by rank order. All mentioned approaches only consider route choice effects in the consequence failure assessment. The consideration of mode and destination choice effects is avoided due to higher model complexity, while the accuracy gain is estimated to be rather small.

\section{METHODOLOGY}

\section{Definition of Vulnerability}

In the field of IMSs, risk is expressed as the probability of occurrence of a certain given event multiplied by the failure probability of a given infrastructure object and the consequences. To integrate natural hazards, both the probability of inadequate performance and the related consequences have to be considered. The consequences of inadequate performance can take two different forms: $(a)$ direct consequences
(CD) to the exposed component in the form of structural damage including repair costs required to return the damaged infrastructure object to its pre-failure state and $(b)$ indirect consequences $(\mathrm{CI})$ to the transportation traffic by restricting or completely denying traffic flow including additional travel time and travel distance costs. The vulnerability of component $i$ is thus the probability of failure due to a given hazard event $\left(P_{f i \mid E}\right)$ multiplied by the sum of the direct and indirect natural-hazard-induced consequences $\left(\mathrm{CD}_{i}, \mathrm{CI}_{i}\right.$ respectively):

$R_{i}=P_{f i \mid E} \cdot\left(\mathrm{CD}_{i}+\mathrm{CI}_{i}\right)$

where



While the methodology to quantify the occurrence probability of hazard events and the direct consequence are presented in Birdsall and Hajdin (19), this paper details a way to quantify the indirect transportation-related consequences of link failures including congestion effects within a national transportation networkwide scale.

\section{Expected Travel Demand Reactions}

Travelers can react to transport infrastructure failure is four ways:

- Detouring the failed link using the remaining link in service,

- Changing the travel mode either for the entire trip-activity chain or parts of it,

- Changing the destination of the activity, and

- Eliminating the scheduled activity and suppressing the trip.

To quantify the CI, each of the mentioned demand reactions requires a different methodology with specific data needs. However, for the case of Switzerland, with its dense road network, it is expected that detours in proximity are available for most of the links. Hence, the additional travel time per traveler caused by a random single link failure is too low to force him or her to change his or her travel mode, the destination of a planned activity, or even to suppress this activity and cancel the travel. This rather strict assumption will be verified for mode choice shifts by comparing the CI per traveler with a threshold describing the inherent cost of a generic mode change. The analysis treated in this paper, however, is confined to the detection of such links. The evaluation of CI involving the mentioned demand responses beyond detours is foreseen for future research.

\section{Assessing Failure-Induced Indirect Transportation Consequences}

The economic evaluation of detour-based CI follows the wellestablished framework of the cost-benefit analysis, which quantifies changes in the transport system in monetary units. This makes the transfer to IMSs rather straightforward, since those systems also use monetary valuation. With the concept of generalized travel costs, the detour-based impact of a link failure can be divided into three parts:

- Additional travel time (TT) costs,

- Additional driving distance costs, and

- Changes in accident rates and associated total accident costs. 
Formally, the additional travel time caused by a link failure is defined as

$$
\Delta \mathrm{TT}_{l}=\sum_{i} \sum_{j \neq i} w_{i j}\left(c_{i j}^{(l)} \cdot c_{i j}^{(0)}\right)
$$

where

$w_{i j}=$ assessed demand weight relation of zone $i$ to zone $j$,

$c_{i j}^{(0)}=$ travel time from zone $i$ to zone $j$ under normal network conditions, and

$c_{i j}^{(l)}=$ travel time from zone $i$ to zone $j$ under modified network conditions with link $l$ severed.

The additional travel distance (TD) caused by a link failure is defined as

$$
\Delta \mathrm{TD}=\sum_{i} \sum_{j \neq i} w_{i j}\left(d_{i j}^{(l)}-d_{i j}^{(0)}\right)
$$

where $d_{i j}^{(0)}$ equals the travel distance from zone $i$ to zone $j$ under normal network conditions and $d_{i j}^{(l)}$ equals the travel distance from zone $i$ to zone $j$ under modified network conditions with link $l$ severed.

The additional accident costs caused by a link failure are defined as

$\Delta \mathrm{AC}=\sum_{m}\left(V_{m, t}^{(l)}-V_{m, t}^{(0)}\right) \cdot \mathrm{ARC}_{t}$

where

$\mathrm{AC}=$ accident costs

$V_{m, t}^{(0)}=$ volume on link $m$ of type $t$ in normal network conditions,

$V_{m, t}^{(l)}=$ volume on link $m$ of type $t$ in network conditions with link $l$ severed, and

$\mathrm{ARC}_{t}=$ accident costs per traffic volume on link of type $t$.

The $\mathrm{CI}$ are then given by

$\mathrm{CI}_{i}=\Delta \mathrm{TT}_{l} \cdot C_{\mathrm{TT}}+\Delta \mathrm{TD}_{l} \cdot C_{\mathrm{TD}}+\sum_{t} \Delta \mathrm{AC}$

where $C_{\mathrm{TT}}$ equals the willingness to pay for travel time reductions and $C_{\mathrm{TD}}$ equals the average cost for driving a defined distance.

These needed values are also commonly used in cost-benefit analysis and are therefore readily available. The relevant figures for Switzerland are the outcome of recent studies (20-22).

Jenelius et al. (15) pointed out that this approach of assessing additional travel time and distance is not applicable when parts of the network are cut off from the rest but lead to unsatisfied demand $(u)$ :

$u_{i j}^{(l)}= \begin{cases}w_{i j} & \text { if } c_{i j}=\infty \\ 0 & \text { if } c_{i j}<\infty\end{cases}$

Here such network failure states are called cut links, because a failure of such a link separates the network into two parts. The economic assessment of the resulting trip suppression is, as already mentioned, beyond the scope of this paper.

\section{APPLICATION}

\section{Network of Scope: Swiss National Transport Model}

All calculations here presented are based on the Swiss national transport model (23), a two-dimensionally constrained disaggregate trip generation, distribution, and mode choice model (24). The Swiss national model is implemented on the basis of 2,949 small zones inside the country and 165 increasingly larger zones beyond the borders of Switzerland. It distinguishes 17 combinations of six trip purposes for three modes (motorized private travel, public transport, and the combined walking and cycling modes). It contains 30,289 undirected links $(19,804$ of which are on Swiss territory) as well as 24,316 nodes (15,405 of which are on Swiss territory). The user-equilibrium assignment model software package VISUM 9.4 (25) was employed.

The network used in the model is a simplification of the complete Swiss transportation network and contains only the relevant links for intercommunal travel demand but not all access roads. Local roads not considered may provide relevant detour alternatives in case of failure of a major road. But because of the small capacity of these access roads with slow speed, this bias is considered small, as validated by Birdsall et al. (26). Nevertheless, in remote places, such as in mountain valleys, these roads have the potential to offer alternatives around cut links that were detected based on the simplified network. Therefore, when links with high failure consequences in these regions are identified, the presence of local alternatives is checked employing Global Positioning System (GPS) navigation networks. In the winter several mountain passes are closed and thus both summer and winter networks have to be considered, representing two cases for the vulnerability of conducting this consequence analysis. The analysis presented in this paper is based on the winter network, since this state is relevant for the major part of the year.

\section{Zero Demand}

According to the definition above, the transport failure consequence measure is only defined for links with assigned demand. Hence, links with zero assigned demand are excluded from this analysis. While it is clear that all links serve some traffic demand, because demand should be very small the failure consequences are also considered to be very small compared with those of links with assigned demand.

\section{Cut Links}

As an initial analysis step, all links whose failure would lead to a cutoff of a network part were detected. This was conducted by the temporary removal of each link and the search of the shortest path the two nodes the link connected before. If no path is found, the link is designated a cut link, which was the case for 1,555 links, while 1,043 of these links have no demand assigned in the model due to spatial aggregation reasons. Otherwise, the length of the shortest path was saved. Subsequently, the presence of bypasses provided by public transport was checked. For that purpose the rail and road networks were merged. For 97 links an alternative path passing through rail links was found. Jelenius (27) assumes that the travel demand is constant over time and that a user wishing to depart during the closure will on average be delayed $\tau=2$ time units, with $\tau$ as the duration of the failure. This approach produces accurate results under the assumption that people do not consider the failure while planning their activities. However, the approach of comparing the two equilibria states (before and after failure) used to assess noncut links assumes that all travelers are aware of the actual network state. Accordingly, the assessment of cut-link failures must focus on the inconvenience of being unable to conduct activities or to at least postpone them. Because assignment models are not suitable to quantify this inconvenience, this cannot be appropriately handled within the general framework presented in this paper and has to be addressed by future research. 


\section{Subnetwork Approach}

The assignment of the Swiss national transport model with a rather loose stop criterion (absolute or relative permitted deviation of impedances $20 / 5 \%$, max relative gap of 0 ), takes around $40 \mathrm{~min}$, depending on the speed of the computer (with an Intel Pentium 4, 3.2 GHZ and $1 \mathrm{~Gb}$ RAM). When multiplied by the total number of links, the calculation of transport failure consequences produced by the independent failure of each link would require 550 days of computation. To reduce computation intensity, knowledge about the characteristics of the transport demand is utilized: because of network hierarchy, the main part of the links serves only a small amount of demand with rather short average path distances. Hence, the redistribution effects in dense parts are assumed to be spatially restricted and these indirect failure consequences can be modeled to a sufficiently accurate level using local subnetworks instead of the whole network. A subnetwork is a limited section of the complete network that is cut out including the internal and the transit demand of this section. To generate the subnetworks, two grid layers with $60-\mathrm{km}$ edge length and an offset of half an edge length in the $x$ - and $y$-axes were overlayed in the Swiss transport model. Afterward, the subnetworks were cut according to the two grids, resulting in 140 subnetworks. The same procedure was repeated using a 30-km grid edge length generating 476 subnetworks. In this way it could be assured that from every link the nearest border of the subnetwork is at least $15 \mathrm{~km}$ and $7.5 \mathrm{~km}$, respectively (for the 30-km edge length subnetworks) afar. As the computational complexity of traffic assignment decreases exponentially with the smaller number of links and zones, the calculation time gains are substantial. The average calculation time for one failure consequence assessment using the 60- and 30-km subnetworks took 35 and $12 \mathrm{~s}$, respectively.

The disadvantage of employing the subnetwork generation is that certain links became cut links within the new subnetwork because the shortest detour extends beyond the boundaries of the subnetwork. Such links, commonly located in the mountainous regions of Switzerland, are characterized by long path lengths and little local density, which also lead to large-scale bypasses. Therefore, two additional subnetworks - one each for the two Swiss mountain ranges (Alps and Jura) - were generated. These mountain range networks were also extended to include parts of the adjacent Swiss plateau, where the main freeways are situated to ensure the shortest paths were included.

To detect how each link is best analyzed, three factors were taken as proxies for the spatial impact of a failure: the normal-state average path length, the link volume, and the shortest bypass of the failed link. Therefore, in addition to recording the shortest bypass and the volume, for each link the mean and standard deviation of the path length were also calculated and recorded. The analysis revealed that links, particularly those ranked high in the function hierarchy such as motorways, exhibited at least two of the three criteria-long path lengths and high demand. In case of failure, severe local congestion was expected. Therefore, the new shortest path for some long-distance origin-destination (O-D) relations may be a large-scale bypass that can only be captured by employing the entire network. Organizationally, the Swiss road infrastructure is classified in national, cantonal, and municipal roads. National roads carry typically high demand with long average path distances. Therefore, this category is assessed with the full network approach. All links that are not part of the national network but which have shortest bypasses longer than $30 \mathrm{~km}$ are assessed with either the Alps or the Jura network. Figure 1 shows the applied methodologies for all links in the considered network and Table 1 summarizes the key figures.

The bulk of the links are assessed with the subnetwork approach, which shows the shortest average bypass distances. The links, ana- lyzed with the full network employed, have, by far, the highest volumes assigned under normal conditions, while the links assessed with the Alps and Jura networks have the longest bypasses. Cut links without rail alternative serve, on the average, only little demand whereas cut links with rail alternative (named Cut Links 2 in Table 1) are similarly characterized as the links assessed with the Alps-Jura networks.

\section{Inelastic Demand}

To reduce computational complexity, only route choice effects are considered within this link failure assessment. Therefore, travel demand is assumed to be inelastic to mode choice and destination choice shifts. This approach has been taken because the link closure duration will be assumed to be long enough that all travelers are likely to be aware of the closed link, and thus a new equilibrium is reached, short enough not to affect mode choice or even destination choice. This assumption should be reasonable for a dense transport infrastructure network as in Switzerland. The assumption of neglecting mode and destination choice shifts is verified by comparison of the average additional user cost per affected traveler with the expected average disutility of any forced mode shift and presented in the section on the relevance of mode choice shifts below.

\section{Rail Infrastructure as Additional Redundancy}

Although demand is assumed to be inelastic, the presence of an alternate mode has to be taken into consideration as this has significant influence on the employed failure consequence assessment. Several valleys in the Swiss mountains have no through access but some are served both by road and rail. In case of a road link failure, the expected demand reaction would be a mode shift from car to rail rather than a change of the destination choice or the suppression of trips, respectively. For the same reasons relevant to the simplified consideration of mode choice shifts, this project currently focuses solely on identifying such links.

\section{RESULTS}

\section{Overview}

Figure 1 presents the magnitude of the indirect failure consequences by line width for all noncut links in the Swiss national model. Links with high volumes (especially highways) and links with long detours (especially in mountainous regions) have the highest impact-the former mainly because of the volume, which has to be redirected; the latter because of the long bypass distances. However, for the majority of the links, their failure induces only minor additional travel times because the volumes are low and the surrounding networks are dense. Early trail runs with rather loose stop criteria (to gain speed) revealed the occurrence of artifacts: links in regions of high travel demand (e.g., agglomeration) far removed from the failed link showing volume changes. To restrict such effects, a stricter stop criterion (absolute or relative permitted deviation of impedances 1.0/1.0\%, max relative gap of $5 \cdot 10^{-5}$ ) was applied, which alleviated these effects at the expense of additional computation time. Nevertheless, for 823 links, all under normal conditions with only small demand assigned, negative additional travel times were obtained. However, it was verified that the negative additional travel times were caused by equilibrium artifacts linked to the stop criterion. 


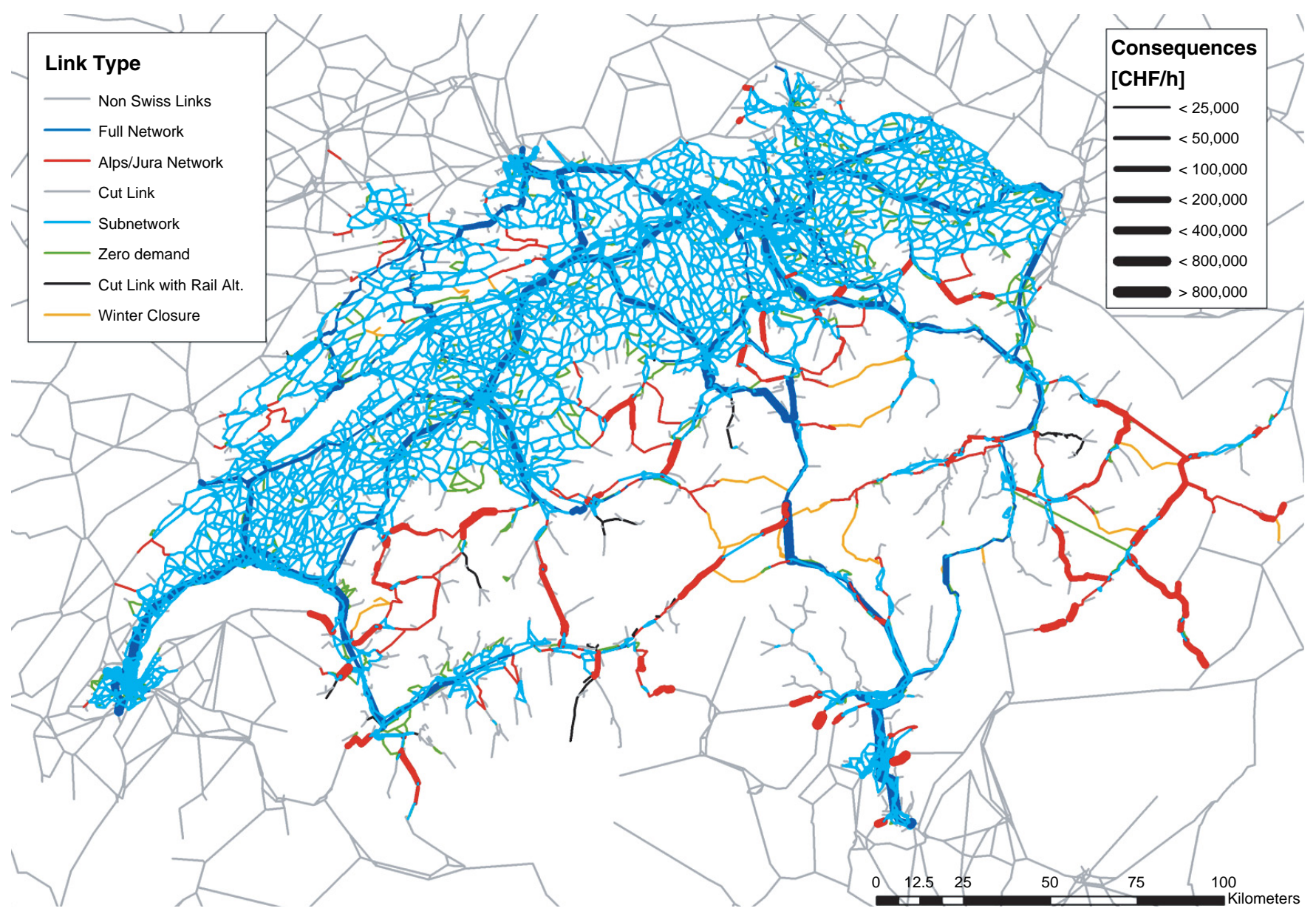

FIGURE 1 Indirect failure consequences (link width) based on Swiss National Transport Model.

The links with the highest values are located in the upper Valais, a mountainous region serviced by a single main valley. In the model, a failure of one of the main valley links cuts this functional region apart. This reveals two critical issues of the applied approach: first, the neglect of low hierarchy links in the assignment model necessitates the inclusion of a post-processing analysis to check for local road link alternatives that are not covered in the simplified network of the national transport model. Second, the assumption of inelastic demand in terms of mode choice is only valid as long the additional travel time or distance for a given person is small. As in the case of the upper Valais, which is also served by rail, one would expect the reaction to be a mode shift toward rail, which would significantly limit the incurred consequences. These local alternative and multimodel post process checks, though not considered were employed to verify the results for the links covered by the Alps-Jura networks. In 120 cases, local bypass links initially not included in the national model were identified by analyzing map data provided by NAVTEQ, normally used for GPSs. For these links the failure consequences measure was

TABLE 1 Assessed Link Characteristics, Separated by Employed Link Analysis Methodology

\begin{tabular}{lcccccr}
\hline & Full Net. $^{a}$ & Alps-Jura & Subnet. $^{b}$ & Cut Links & Cut Links 2 & No Dem. $^{c}$ \\
\hline Number of assessed links & 1,243 & 689 & 14,613 & 1,555 & 97 & 1,976 \\
Average volume & $12,263.7$ & $1,460.4$ & $2,468.7$ & 245.5 & $1,615.7$ & 0 \\
Std dev. volume & $9,634.8$ & $1,395.4$ & $2,851.3$ & 965.9 & 165.8 & - \\
Average path distance & 62.7 & 46.5 & 21.4 & 6.89 & 32.2 & - \\
Std. dev. path & 41.8 & 25.5 & 13.5 & 16.5 & 14.1 & - \\
Average detour length & 8.12 & 85.5 & 6.5 & - & $(7.7)$ & 9.9 \\
Std. dev. detour & 11 & 53.7 & 5.4 & - & $(4.5)$ & 22.7 \\
\hline
\end{tabular}

${ }^{a}$ Net. $=$ network.

${ }^{b}$ Subnet. = subnetwork.

${ }^{c}$ Dem. $=$ demand. 
recalculated, and the result was substantially smaller values; 108 links were detected to have a rail alternative with sufficient capacity and omitted for further analysis.

\section{Highest Indirect Consequences}

Finally, the most exposed link in terms of additional travel time is the motorway section passing Monte Ceneri, which serves as a main European north-south corridor. The only available alternative within reasonable distance is an old trunk road whose capacity is too small to carry the rerouted traffic with an adequate service level. Its failure would induce additional travel time per day of $36,194 \mathrm{~h}$, additional mileage of $344,310 \mathrm{~km}$ which, together with the increasing accident cost, sums up to indirect failure consequences of 1.57 Mio Swiss francs (CHF)/day. The Gotthard north-south Alp tunnel accounts for a loss of $0.79 \mathrm{Mio} \mathrm{CHF} /$ day but entails an additional vehicle distance of $1.01 \mathrm{Mio} \mathrm{km}$, the highest detour mileage of all failure scenarios. For 18 of the 20 most severe cases, a rail alternative is also present, which suggests that the calculated value should be considered as upper limit, since mode choice shifts were excluded from the analysis.

The distribution of the indirect consequences shows exponential behavior (Kolmogorov-Smirnov test statistic of 43.7) with a $\lambda$-parameter of $43.7 \cdot 10^{-5}$. This reveals that only a small number of link failures lead to substantial losses, while for most links the losses are less significant.

\section{Congestion Effects}

The additional travel distances are correlated with the additional travel time, especially for links in less dense parts of the network where the additional travel time is mainly determined by the bypass length rather than influenced by local congestion. Hence, the correlation for links assessed in the Alps-Jura networks or in subnetworks is substantially higher than for the highway links that were assessed with the full network (Pearson coefficient $0.81 / 0.70$ to 0.53 , all highly significant at the 0.01 level). Dividing the additional distance by the additional travel time yields an indicator of the congestion effects caused by the link failure.

It is assumed that for scenarios with this indicator below $50 \mathrm{~km} / \mathrm{h}$, congestion on detours is relevant since the slowest attributed free-flow speed in the network is $50 \mathrm{~km} / \mathrm{h}$ (except in some inner-city links). Overall, in $12.4 \%$ of the cases, this condition is fulfilled and potential congestion effects are relevant.

\section{Accuracy of Subnet Approach}

The failure consequences of the majority of the links are assessed with the $60-\mathrm{km}$ subnetwork methodology. As this approach might lead to systematic bias, it is important to check that the failure consequences agree with the full network-assessed consequences. For this reason, all links subject to the full and Alps-Jura networks approach were also assessed with the 30- and 60-km subnetworks.

Figure 2 shows a scatterplot comparison of the subnetwork and the full and the Alps-Jura networks-assessed consequences. Eight outliers from the 30-km subnetwork are not included since these values extend beyond the scale of the figure. Except for some cases, the results calculated by the 30 - and $60-\mathrm{km}$ subnetworks are very consistent with the full-network results. The actual assessed consequence level is in these cases overestimated because the limited network does not cover all relevant bypasses. The small number of outliers and the low deviation



FIGURE 2 Comparison of additional user costs (accident cost not considered) between 30-km and $60-\mathrm{km}$ subnetwork against full and Alps-Jura networks. 
from the reference values confirm that demand shifts are normally spatially very restricted. This is mainly a product of the high network density and the short average path distances, which automatically restrict the spatial scale of the analysis. The outliers are the links representing the motorway leading through Ticino, a region that is characterized by steep mountains where only one relevant nearby detour is available. This detour cannot absorb all of the demand, which is rerouted to more distant detours that are not included in the subnetworks.

\section{Relevance of Mode Choice Shifts}

This paper establishes an additional user cost mode choice shift threshold and identifies the conditions where such a threshold is breeched. Such an approach is clearly a simplification since the additional user costs of each scenario follow a distribution across all users. However, the objective of this paper was only to establish an initial assessment of the relevance of possible mode choice effects and where such an approach should influence the assessment of the network. To detect condition states that might induce destination choice shifts is not feasible with this methodology since much more detailed information about alternative destinations and their respective attributes would be necessary. To obtain a conservative threshold of additional user costs (indirect consequences without accident cost), the following additional elements of generalized cost are set up:
- Access and egress time of $10 \mathrm{~min}(10 \mathrm{~min} * 0.38 \mathrm{CHF} / \mathrm{min})$,

- 0.5 transfers $(0.5 * 2.34 \mathrm{CHF} /$ transfer $)$,

- Transfer time of $5 \min (5 \mathrm{~min} * 0.5 * 0.10 \mathrm{CHF} / \mathrm{min})$.

The valuations of the access and egress time as well as transfers are based on VSS (20). Figure 3 identifies all links whose failure causes an increase of average additional user cost per affected traveler above the threshold of 5.25 CHF.

Overall, 923 links might be subject to mode choice shifts. However, this has to be put into perspective: First, the threshold with 10-min access-egress time and 0.5 transfers with 5 min transfer time is intentionally low. Additionally it can be assumed that for most demand relations the travel time by car is substantially shorter than by public transport. Second, the analysis does not currently consider the possibility of a relevant public transport detour.

Even if there is a rail alternative available, the impact of mode choice shifts on the CI can be relatively low, as the following case study shows [for more details see Birdsall et al. (26)]: For the failure of the Gotthard road tunnel, O-D-specific mode choice effects were calculated using a logit model with the same parameters used in the national transport model. Although the average failure-induced additional user costs were 40 CHF per person, only for $10 \%$ of the demand mode shifts were expected. Interestingly, the indirect failure consequences decreased by $10 \%$. It is clear that this finding cannot be generalized and applied to other links. However, it shows that

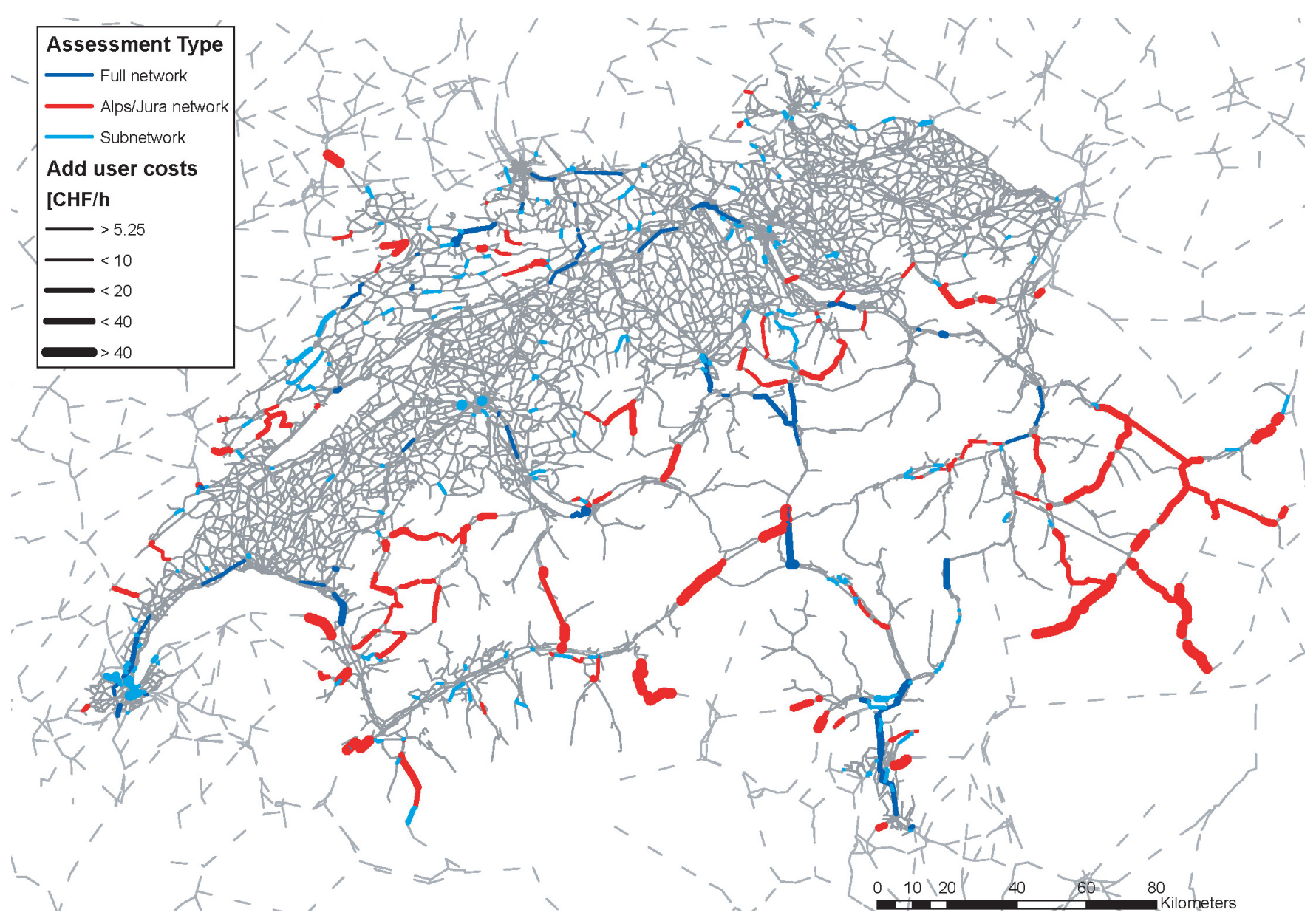

FIGURE 3 Relevance of mode choice: links with average additional user costs higher than 5.25 CHF. 
the overestimation of the indirect failure consequences due to the neglect of mode choice effects is moderate.

\section{MODELING INDIRECT FAILURE CONSEQUENCES}

Using the subnetwork approach, computation time to assess indirect failure-induced traffic consequences on a networkwide scale became reasonable. For the Swiss case, it takes around 5 weeks on a Pentium 4, 3.2GHz Processor with 1Gb RAM. However, knowledge of script programming and the availability of an assignment model are required to generate subnetworks. Hence, it would be desirable to assess traffic consequences more directly. One approach would be to use a statistical model to estimate a link's indirect failure consequences. The statistical model combines spatial data and information on the link volume and estimates consequence influence coefficients for each parameter. Using the resulting parameters, one can indicate the magnitude of vulnerability without an assignment model.

Since the consequence measure follows an exponential distribution, the employed statistical model is a generalized linear model that assumes the dependent variable to be exponentially distributed. The independent variables to determine the failure consequences were chosen to match preferably little input data requirements, since they should serve in cases with no assignment model available. The assignment-based results presented above showed large failure consequences for links with high-volume, long-detour distances or minimal network density. Therefore, the presented statistical model employed these factors but no data, which can only be derived from assignment models like, for example, the average path distance. As categorical variable, the network type with which each link's failure consequences were calculated was also included in the model. This additional information can also be seen as a proxy for the average path distance as shown in Table 1, but requires no assignment model-derived information. The data basis is built with positive values of additional travel time and indirect failure consequences.

Since in the calculation of the failure consequences the volume acts as a multiplying factor, this variable was modeled as an interaction term. The categorical variables were employed as dummy variables in order to estimate separate parameters for links according to the network type used in the assessment of prior consequence. The final model has the following structure:

$$
\begin{aligned}
\mathrm{CI}_{i}= & \text { const }+\beta_{\mathrm{TTD}, \mathrm{NT}_{m}} \cdot \text { dummy }_{\mathrm{NT}_{m}, i} \cdot V_{i} \cdot \mathrm{TTD}_{i} \\
& +\beta_{D, \mathrm{NT}_{m}} \cdot \text { dummy }_{\mathrm{NT}, i} \cdot\left(\frac{V_{i}^{2}}{C_{5 \mathrm{~km}, i}}\right)
\end{aligned}
$$

where

$$
\begin{aligned}
\mathrm{CI}_{i}= & \text { indirect failure consequences }[\mathrm{CHF} / \mathrm{day}], \\
\mathrm{const}= & \text { constant } \\
\beta_{\mathrm{TTD}, \mathrm{NT}_{m}}= & \text { parameter estimate "detour travel distance" for } \\
& \text { netwrok type } m, \\
\text { dummy }_{\mathrm{NT}_{m}, i}= & \text { dummy network type used for the assessment, } \\
V_{i}= & \text { volume }[\mathrm{veh} / \text { day }] \\
\mathrm{TTD}_{i}= & \text { travel time shortest detour }[\mathrm{h}], \\
\beta_{D, \mathrm{NT}_{m}}= & \text { parameter estimate "network destiny" for network } \\
& \text { type } m, \text { and } \\
+C_{5 \mathrm{~km}, i}= & \text { capacity } \cdot \text { length of all links within a radius of } \mathrm{km} \\
& \text { [veh } \cdot \mathrm{km}] .
\end{aligned}
$$

Whereas the first term accounts for the shortest detour, the second term captures capacity limitation effects. During the modeling process, other variables were tested but this model proved to have the highest
TABLE 2 Generalized Linear Model of Indirect Failure Consequences

\begin{tabular}{lrrr}
\hline Parameter & \multicolumn{1}{c}{$\beta$} & Std. Error & Wald $\chi^{2}$ \\
\hline Const & 376.90 & 163.20 & 5.33 \\
Alp-Jura $\cdot V_{i} \cdot \mathrm{TTD}_{i}$ & 28.98 & 0.75 & $1,012.45$ \\
Full $\cdot V_{i} \cdot \mathrm{TTD}_{i}$ & 28.76 & 0.75 & 314.19 \\
Subnet $\cdot V_{i} \cdot \mathrm{TTD}_{i}$ & 14.90 & 0.83 & 323.20 \\
Full $\cdot V_{i}^{2} / \mathrm{C}_{5 \mathrm{~km}, i}$ & 0.84 & 0.07 & 153.84 \\
Subnet $\cdot V_{i}^{2} / \mathrm{C}_{5 \mathrm{~km}, i}$ & 0.63 & 0.04 & 272.21 \\
\hline
\end{tabular}

explanatory power while remaining simple in its structure. The results presented in Table 2 affirm the earlier statement that demand shifts are assumed to be spatially restricted, as the included variables describe only the adjacent network part of the given link.

Overall, the importance of the shortest detour characteristics is obvious. The term describing capacity limitation effects for links assessed with the Alps-Jura networks is omitted since its effect is insignificant. For links assessed with the full network, this term has a higher impact than for subnetwork links because congestion effects seem to be more crucial in case of failure of a motorway link. The distribution of the error term is normal (Kolmogorov-Smirnov test statistic of 43.1), fulfilling the need of the estimation methodology. Further analysis of the error term showed that the model indeed is able to predict the effective failure consequences for cases with low or middle failure consequences but systematically underestimated the cases with the highest values. These links are characterized by the presence of only one nearby relevant detour whose capacity is too little to absorb the rerouted demand. This specific characteristic is not represented adequately by the independent variables. These links, however, can easily be identified by checking the presence of local alternatives for those small numbers of links with high predicted failure consequences.

\section{CONCLUSION}

This paper presents a new approach for calculating transport-related failure consequences including congestion effects across a national network. Failure consequences calculated using subnetworks compared against those using the full network are very accurate and reliable since the rerouting effects tend to be spatially restricted around the failed link. Although it was expected that the spatial impact of a failure increases with longer path distances and shortest detour lengths, the results of the subnetwork approach are also very stable when measured against these two factors. Only a few links required the use of the full or the Alps-Jura networks.

Furthermore, by applying this analysis one can identify the links where the traffic failure consequence is influenced by congestion. It becomes obvious that congestion effects influence the result substantially for a minor part of link failures only. However, the majority of consequences are caused by congestion effects, since in these cases the costs of the additional travel time tend to be substantially higher compared with the costs caused by the additional travel distance. Using the statistical model presented in this paper, one could attempt to predict which cases would require the inclusion of congestion effects in advance, thereby saving additional computation time.

Finally, application of this methodology it demonstrates how network structure influences failure consequences. These findings are especially valuable if no assignment model is available or if one is interested in identifying the links with the potentially largest traffic 
consequences for future detailed analysis. The methods used in the paper do have some limitations, which are potential topics of future research. The inclusion of mode choice shifts could increase the accuracy of the methodology, especially in Switzerland with its dense rail network and the mountainous landscape that causes long detours. For this purpose, the modal split had to be recalculated using the actual user costs within the failure scenario. The inclusion of destination choice effects necessitates recalculation of the trip distribution. Since both tasks are computation intensive but have a relevant effect only for a restricted number of links, it would be interesting to be able to forecast the need for the inclusion of these effects according to readily available proxies such as network density, availability of other modes, and other relevant destinations, as well as characteristics of the flow bundle.

Following the analysis of the initial network, links with local alternatives were re-analyzed to determine if any local, not yet considered link alternatives existed. However, this check was only implemented for links with high indirect consequences or those situated in mountainous regions with long detours. It might be interesting to quantify indirect consequences using a full representation of the actual available road links in order to quantify the effects of the additionally provided capacity and detour possibilities.

In this study each failure is assumed to be mutually exclusive. This assumption is - at least for certain types of natural hazards such as floods, avalanches, and torrents-not reasonable. Since the calculation of all possible link failure combinations is not feasible, it would be only reasonable to calculate joint failure vulnerabilities if a certain probability of this jointness could be derived from the analysis of the hazard maps, which is not available at the moment. One potential approach would be to use genetic algorithms to identify link failure combinations, which induce the most severe consequences as upper limits.

\section{ACKNOWLEDGMENT}

The authors gratefully acknowledge that this research was made possible through a grant from the Swiss National Science Foundation National Research Program 54: Sustainable Development of the Built Environment.

\section{REFERENCES}

1. Thompson, P., E. Small, M. Johnson, and A. Marshall. The Pontis Bridge Management System. Structure Engineering International, Vol. 8, No. 4, 1998, pp. 303-308.

2. Hajdin, R. KUBA Version 4.0. Presented at Conference on Operation and Maintenance and Rehabilitation of Large Infrastructure Projects, Bridges, and Tunnels, Copenhagen, Denmark, May 2006.

3. Tyagunov, S., P. Heneka, L. J. Zschau, B. Ruck, and C. Kottmeier. CEDIM: From Multi-Hazards to Multi-Risks. Presented at Amonia Conference, Dec. 2005. http://edim.de/english/904.php.

4. King, C., and R. Bell. Technologies and Trends for Disaster Monitoring and Reduction. Presented at Earthquake Engineering in the 21st Century (EE-21C) Conference, Skopje, Macedonia, Aug. 2005

5. FEMA. Using Hazus-MH for Risk Assessment. 2004.

6. Schneider, S. H., S. Semenov, A. Patwardhan, I. Burton, C. Magadza, M. Oppenheimer, A. B. Pittock, J. B. Smith, A. Suarez, and F. Yamin. Assessing Key Vulnerabilities and the Risk from Climate Change. In Fourth Assessment Report of the Intergovernmental Panel on Climate Change (M. L. Parry, O. F. Canziani, J. P. Palutikof, P. J. van der Linden, and C. E. Hanson, eds.), Cambridge University Press, Cambridge, Mass., 2007, pp. 779-810.

7. Berdica, K. An Introduction to Road Vulnerability: What Has Been Done, Is Done, and Should Be Done. Transport Policy, Vol. 9, No. 2, 2002, pp. 117-127.

8. Taylor, M. A. P., and G. M. D’Este. Network Vulnerability: An Approach to Reliability Analysis at the Level of National Strategic Transport Net- works. In Network Reliability of Transport (M. G. H. Bell and Y. Iida, eds.), Pergamon, Oxford, United Kingdom, 2003, pp. 23-44.

9. Knnop, V. L., S. P. Hoogendoorn, and H. J. van Zuylen. Quantification of Impact of Spillback Modeling in Assessing Network Reliability. Presented at 86th Annual Meeting of the Transportation Research Board, Washington, D.C., 2007.

10. Matisziw, T. C., A. T. Murray, and T. H. Grubesic. Evaluating Vulnerability and Risk in Interstate Highway Operation. Presented at 86th Annual Meeting of the Transportation Research Board, Washington, D.C., 2007.

11. Bell, M. G. H. A Game Theoretic Approach to Measuring Performance Reliability of Transport Networks. Transportation Research Part B: Methodological, Vol. 34, No. 6, 2000, pp. 533-545.

12. Bell, M. G. H., and C. Cassir (eds.). Reliability of Transport Networks, Research Studies Press, Baldock, Hertfordshire, United Kingdom, 2001.

13. Chen, A., H. Yang, H. K. Lo, and W. H. Tang. A Capacity-Related Reliability for Transportation Networks. Journal of Advanced Transportation, Vol. 33, No. 2, 2007, pp. 14-158.

14. Yida, Y., and H. Wakabayashi. An Approximation Method of Terminal Reliability of Road Network Using Partial Minimum Path and Cut Sets. Transport Policy, Management, and Technology Toward 2001: Selected Proceedings of the Fifth World Conference on Transport Research, Western Perodicals, Ventura, Calif., 1989, pp. 367-380.

15. Jenelius, E., T. Petersen, and L.-G. Mattsson. Importance and Exposure in Road Network Vulnerability Analysis. Transportation Research Part A: Policy and Practice, Vol. 40, No. 7, 2006, pp. 537-560.

16. Knoop, V. L., M. Snelder, and H. J. van Zuylen. Comparison of LinkLevel Robustness Indicators. Presented at 3rd International Symposium on Transportation Network Reliability, Delft, Netherlands, July 2007.

17. Scott, D. M., D. C. Novak, L. Aultman-Hall, and F. Guo. Network Robustness Index: A New Method for Identifying Critical Links and Evaluating the Performance of Transportation Networks. Journal of Transport Geography, Vol. 14, 2006, pp. 215-227.

18. Wardrop, J. G. Some Theoretical Aspects of Road Traffic Research. Proceedings of the Institution of Civil Engineers, Part II, Vol. 1, No. 36, 1952, pp. 352-362.

19. Birdsall, J., and R. Hajdin. Vulnerability Assessment of Individual Infrastructure Objects Subjected to Natural Hazards. Presented at 10th International Bridge and Structure Management Conference, Buffalo, N.Y., Oct. 2008.

20. VSS Kosten-Nutzen-Analysen (KNA) bei Massnahmen im Strassenverkehr, Zeitkosten im Personenverkehr. Technical Report, SN 641 822a, Swiss Association of Road and Transport Professionals (VSS), Zurich, 2009.

21. VSS Kosten-Nutzen-Analysen im Strassenverkehr, Unfallkostenraten und Unfallkostenziffern. Technical Report, SN 641 824, Swiss Association of Road and Transport Professionals (VSS), Zurich, 2008.

22. VSS Kosten-Nutzen-Analysen (KNA) bei Massnahmen im Strassenverkehr, Betriebs-und Unterhaltskosten von Strassenfahrzeugen. Technical Report, SN 641 827, Swiss Association of Road and Transport Professionals (VSS), Zurich, 2009.

23. Vrtic, M., P. Fröhlich, N. Schüssler, S. Dasen, S. Erne, B. Singer, K. W. Axhausen, and D. Lohse. Erzeugung neuer Quell-/Zielmatrizen im Personenverkehr. Technical Report, Swiss Federal Department for Environment, Transport, Energy, and Communication, Swiss Federal Office for Spatial Development, Swiss Federal Roads Authority and Swiss Federal Office of Transport, IVT, ETH Zurich, Emch, und Berger, Institute for Transportation Planning and Traffic, Technical University Dresden, Zurich, 2005.

24. Vrtic, M., D. Lohse, P. Fröhlich, C. Schiller, N. Schüssler, H. Teichert, and K. W. Axhausen. Simultaneous Two-Dimensionally Constrained Disaggregate Trip Generation, Distribution, and Mode Choice Model: Theory and Application for a Swiss National Model. Transportation Research Part A: Policy and Practice, Vol. 41, No. 9, 2007, pp. 857-873.

25. TV Benutzerhandbuch VISUM 9.4. Traffic Mobility Logistics (PTV), Karlsruhe, Germany, 2006.

26. Birdsall, J., A. Erath, R. Hajdin, and K. W. Axhausen. Consideration of Vulnerability in the Management of Swiss Transportation Infrastructure. Technical Report. National Research Programme 54: Sustainable Development of the Built Environment, Swiss National Science Foundation, Bern, 2009.

27. Jenelius, E. Network Structure and Travel Patterns: Explaining the Geographical Disparities of Road Network Vulnerability. Journal of Transport Geography, Vol. 17, No. 3, 2009, pp. 234-244.

The Critical Transportation Infrastructure Protection Committee sponsored publication of this paper. 